\title{
Pilot Assisted Channel Estimation in MIMO-STBC Systems over Time-Varying Fading Channels- A Review
}

\author{
Manisha Patel \\ M.Tech Scholar, Oriental \\ College of Technology \\ (OCT) Bhopal, India
}

\author{
Rovin Tiwari \\ Assistant professor, \\ Oriental College of \\ Technology (OCT) Bhopal, \\ India
}

\begin{abstract}
In this paper, challenge about the condition of channel state info (CSI) in multiple-input multiple-output (MIMO) systems supported space time block codes (STBC) over slow timevarying Rayleigh attenuation channels are addressed. We develop a novel MIMO control assessment algorithmic rule that adopts a pilot symbol assisted modulation (PSAM) which has been proved to be effective for attenuation channels. In this approach, pilot symbols are periodically inserted into the information stream that's sent through the orthogonal STBC encoder. At the receiver, we propose a simple MIMO channel estimation methodology before getting used by STBC decoder. Simulation results indicate that the proposed pilotassisted MIMO conception provides accurate channel estimates. The impact of Doppler frequency on performance scheme is also investigated by simulation.
\end{abstract}

\section{Keywords}

Multiple-input-multiple-output (MIMO), pilot assisted systems, rate distortion. Channel Estimation.

\section{INTRODUCTION}

The escalating demands for speedy and reliable wireless communications have spurred development of multiple input-multiple-output (MIMO) systems with multiple antennas at each transmitter and receiver sides. To efficiently harvest the capability and variety gains obtainable by MIMO channels, a number of space-time continuum process techniques have been developed, such as Bell Labs layered space-time continuum architectures and orthogonal spacetime continuum block codes, to name a few. To further enhance the system capability, info-theoretic research indicates that a feedback channel can be utilized to provide channel state information (CSI) to the source aspect, which could have an effect on closed-loop capability gains significantly once the coherence time of the MIMO channel is sufficiently large. When excellent feedback of CSI is unavailable due to complexness or utility constraints, the performance limits of MIMO systems under noisy or quantized feedback are evaluated in the study. A number of feedback techniques are devised to understand the close-loop capability gain. In power-control schemes based on quantized feedback info are designed to reduce an upper bound of multiple-input- single-output (MISO) system. At what time only the index of the most excellent beam forming vector is fed back to the transmitter, the problem of quantized most signal-to-noise ratio (SNR) beam forming is solved within the study. Under feedback-link ability constraint, a hybrid beam forming and adaptive power-control architecture is developed in the study. For scalar power feedback and per-antenna vector power feedback, the problem of optimal MIMO link capacity is calculated in the study, while the problem of optimal MIMO multiuser design is solved in the study in. Recently, two detailed form of fractional feedback, specifically, channel mean feedback (CMF) and channel covariance feedback (CCF), include be investigate for slowvarying and quickly varying MIMO channels, respectively. Based on CMF, optimal multiantenna broadcast precoder plan has been pursue in the study, while with $\mathrm{CCF}$, a required and adequate condition for the optimality of ray form is obtained in the study. The error performance of adaptive modulation with delayed response, minimum mean square error (MMSE) channel forecaster, and broadcast beam forming is investigated in the study. According to the literature, the pilot symbol assisted modulation has recently emerged as a promising MIMO estimator used for time-varying wireless communication systems. It offers satisfactory presentation with practical computing complexity. Therefore, the use of PSAM come close to perform channel inference is recommended here for practical setting. In this effort, a performance analysis of the novel pilot symbol assisted modulation system working on MIMO channels and TCMSTBC codes are explored. Here are two generally classified TCM categories: the predictable TCM and the multidimensional trellis coded modulation which is known to provide high bandwidth efficiency. In this paper, we focus on the performance analysis of the second kind of TCM as an outer code. We study here on the recognized four multidimensional TCM (4D-TCM) scheme described in which offers high data rate. A symbol interleaver is well introduce in the program chain in arranges to cut the effect of burst error due to fading. The channel characteristics are assumed to be sluggish fading and steady over the STBC secret word period. The received pilot signals give information regarding the MIMO channel as predictable by the novel channel estimation before being interpolated. Simulation results confirm the efficiency of the projected system which show good bit error rate (BER) performance as compared to the perfect coherent system with no excessive complexity growth. Channel estimation is an important job in wireless communication systems. it can be done in two ways: (1) introduction the pilot tones into all of the sub carriers of OFDM symbols with certain period, or (2) introduction the pilot tones into each OFDM symbol. The data acknowledged is in form of MIMO Channel Matrix. This matrix can be determined using statistical, measurement base or site-specific deterministic technique. In this paper, we present a broad review of enormously important researches existing in the literature for channel estimation in MIMO-OFDM system, by using diverse methods. The review researches are classifying with the type of channel estimation methods.

\section{RELATED WORKS}

A wide range of research methodologies is employed for channel estimation in MIMO-OFDM is presented here. The reviewed works are classify the different channel estimation methods like pilot based, blind channel, LMS and RLS, LS \& MMSE and other channel estimation methods. 
Emna Ben Slimane et. al. [1] " Pilot Assisted Channel Estimation in MIMO-STBC System Over Time-Varying Fading Channels" In this projected work challenges about the provision of channel state information in multiple-input and multiple output (MIMO) system base on space time block codes (STBC) on slow time-varying Rayleigh fading channels are address. We developed a novel MIMO channel estimation algorithm to adopt a pilot symbol assisted modulation (PSAM) which has been proven to be effectual for fading channels. In this advance, pilot symbols are time to time inserted into the data stream that is sent by the orthogonal STBC encoder. At the receiver point, we intend a basic MIMO channel estimation method previous to being used by STBC decoder. correct and straightforward PSAM estimation method is anticipated for MIMO based on orthogonal STBC codes. The transmitter just insert known optimally and equally paced pilot symbol in data information block. The join signal is coded by using orthogonal STBC code. The transmitted signal is corrupted by additive noise and slow fading. The slow fading channel is modeled by the Jakes model; it is also chosen to be steady over the STBC codeword phase. The receiver estimate and interpolates the channel capacity provided by pilot symbols in order to attain the amplitude and the phase reference for detection. Simulation result show that channel estimation base on PSAM technique is correct in terms BER for both MIMO schemes. The advantage of this technique is its achievement ease in addition to the competitive performance. It is also shown that estimation method is right for slow time-varying fading channels and can be extended to fast time-varying fading channels.

Duo Zhang, et. al. [2] " On the limits of Feedback Rates for Pilot-Assisted MIMO Systems" For pilot-assist multipleinput-multiple-output (MIMO) system with inadequate feedback, we examine the relationship in between the feedback transmission rate and resulting gain of closed-loop capacity. Based on this relationship quantify by rate-distortion theory, we examine the upper and lower bounds of the feedback rate that would affect gain of positive closed-loop capacity without excessively consuming feedback transmission resources. widespread simulations are carry out to authorize the analytic result and to shed light on the attainable closed-loop MIMO capacity given the system design parameterized by the number of antennas, pilot power allotment, transmit signal-to-noise ratio, and finite feedback rate. These results provide guideline on the feedback channel for significant designs of useful pilot-assisted MIMO systems. For practical communication design with pilot-assisted MIMO transceivers and CSI feedback, we have conduct analysis and simulations to address the impact of feedback rates on closedloop feedback gains. Given the system parameters including the number of antennas, pilot power allocation and transmit SNR, we have to derive the mathematical relationship connecting feedback rate to closed-loop capacity gain, which in turn leads to the lower and upper bounds of the feedback rate for taking up in practical implementations.

Jyoti P. Patra et. al. [3] “ A Comb-Type Pilot Symbol Aided Channel Estimation for the STBC based OFDM System over Frequency Selective Channel" In this paper, the channel estimation method for space time block code (STBC) based on multi-input multi-output (MIMO) orthogonal frequency division multiplexing (OFDM) is investigate for multipath fading channels. The channel estimation is based on combtype pilot arrangement where' $s$ the pilot subcarriers are placed at difine interval of each OFDM symbol block and are orthogonal between differ transmitting antennas because the advantages of orthogonal pilot subcarrier, the real transmitted signal is completely recover from the mixed transferred signal on the receiver side. The least square algorithm is adopted for assessment of channel at pilot subcarrier while the interpolation is completed at data subcarrier. The performance of the develop channel estimation method is compare with the recognized single-input single-output (SISO) OFDM system based on symbol error rate (SER). The simulation results shows that the STBC OFDM gives improved performance than SISO OFDM and the SER decreases with the signal to noise ratio (SNR) and the number of receive antenna increases. Pilot aided channel estimation technique using comb type pilot arrangement with different interpolation method for STBC based OFDM system is investigated $\mathrm{w}$ multipath fading channel. The orthogonal properties of the pilot arrangement have been described in a great detail. Due to the orthogonal pilot arrangement between the two transmitting antennas, the channel estimation technique is simple and low computational cost as no matrix inversion is required. The simulation results shows that comb type pilot with spline and low-pass interpolation technique of STBCOFDM system performs better than the conventional OFDM system. From the simulation result, it is clear that low-pass interpolation outperform than spline type interpolation method.

Mohamed Marey, et. al [4] "Blind STBC Identification for Multiple-Antenna(MA) OFDM Systems" The problem of space time block identification for multiple-antenna orthogonal frequency-division multiplexing (FDM) systems operating over frequency-selective channels for the first time in literature. Previous investigations available on the topic of STBC identification were limited to single-carrier systems operating on frequency-flat channels. OFDM systems make this subject more challenging to handle since the identifiers works in frequency-selective channels with little or without knowledge of the beginning of the OFDM blocks, frequencyselective channel coefficients and OFDM parameters, by taking advantage of the space time redundancy we show that, STBC detection can be performed by exploiting the crosscorrelation of the signals which receive from different antennas with a discriminating feature. A novel STBC-OFDM detection method is projected. Analytic investigation and result from simulation experiment point out that the crosscorrelation of the received signals from differ antennas depend on the transmitted STBC. Based on statistical properties of received signals, we intend a new hypothesis test for STBC identification. The proposed technique does not require information about channel coefficients, modulation of the received signal, signal-to noise ratio, or starting time of the OFDM and STBC blocks. Simulation results show satisfactory identification accuracy in the presence of clocktiming offset, spatially correlated noise, and correlated channel. The principal shortcoming of the projected method, its sensitivity to carrier frequency offset, will be priority in the further development of the method.

Leandro D' Orazio, et.al.[5] “ MMSE Multi User Detection with GA-assisted Channel Estimation for STBC MC-CDMA Mobile Communication Systems" MIMO MC-CDMA technique have been planned in order to increase system capacity through frequency-selective wireless channels. The key characteristic of MIMO MC-CDMA is the potential of exploiting range jointly in time, space and frequency domains. In particular, Alamouti' s coding scheme has been projected as a very simple and scientifically elegant tool in order to put into action space-time block coding. The main difficulty to be 
addressed is to efficiently recombine variety in order to achieve the best performance in the presence of multi-user interference. In this paper, we are proposed a linear multi-user receiver based on the MMSE criterion of low-complexity supported by a Genetic-Algorithm assisted MMSE channel estimation. Numerical result show performances very near to MMSE MUD exploiting ideal knowledge of channel position information, thus confirming the near-optimal behavior of genetic algorithms in the process of signal detection and equalization. In projected model discussion is based on a novel Gaassisted MMSE channel estimation method for STBC MC-CDMA system working in time-varying multi path fading channels. Advance on, the achieved estimated channel matrix have been employed to implement the canonical MMSE Multi- User Detection. The analysis of the BER performances yielded by the MMSE-MUD with the ideal and not-ideal CSI knowledge evidenced that the proposed GAassisted channel estimation allow systems to reach performances very close to those which achieved in the ideal case, thus confirming the already claim quasi-optimal behaviors of genetic algorithms when apply to solve telecommunications problems.

\begin{tabular}{|c|c|c|c|}
\hline $\begin{array}{l}\text { Citatio } \\
\text { n }\end{array}$ & Title & $\begin{array}{l}\text { Journal } \\
\text { Name/Year }\end{array}$ & $\begin{array}{l}\text { Research } \\
\text { scope }\end{array}$ \\
\hline [1] & $\begin{array}{l}\text { Pilot } \\
\text { Assisted } \\
\text { Channel } \\
\text { Estimation } \\
\text { in MIMO- } \\
\text { STBC } \\
\text { System } \\
\text { Over Time- } \\
\text { Varying } \\
\text { Fading } \\
\text { Channels }\end{array}$ & $\begin{array}{l}\text { IEEE } \\
\text { International } \\
\text { Workshop on } \\
\text { Resource Allocation } \\
2014\end{array}$ & $\begin{array}{l}\text { In it a } \\
\text { accurate and } \\
\text { straightforw } \\
\text { ard PSAM } \\
\text { estimation } \\
\text { method is } \\
\text { proposed for } \\
\text { MIMO } \\
\text { based on } \\
\text { Orthogonal } \\
\text { STBC codes. }\end{array}$ \\
\hline [2] & $\begin{array}{l}\text { On the } \\
\text { limits of } \\
\text { Feedback } \\
\text { Rates for } \\
\text { Pilot- } \\
\text { Assisted } \\
\text { MIMO } \\
\text { Systems }\end{array}$ & $\begin{array}{l}\text { IEEE } \\
\text { TRANSACTI-ONS } \\
\text { ON VEHICULAR } \\
\text { TECHNOLOGY } \\
\text { June } 2007\end{array}$ & $\begin{array}{l}\text { In this } \\
\text { results } \\
\text { provide } \\
\text { guideline on } \\
\text { the feedback } \\
\text { channel for } \\
\text { meaningful } \\
\text { designs of } \\
\text { practical } \\
\text { pilot-assisted } \\
\text { MIMO } \\
\text { systems. }\end{array}$ \\
\hline [3] & $\begin{array}{l}\text { A Comb- } \\
\text { Type Pilot } \\
\text { Symbol } \\
\text { Aided } \\
\text { Channel } \\
\text { Estimation } \\
\text { for the } \\
\text { STBC } \\
\text { based }\end{array}$ & $\begin{array}{l}\text { IEEE Asia Pacific } \\
\text { Conference } \\
2013\end{array}$ & $\begin{array}{l}\text { In it comb } \\
\text { type pilot } \\
\text { arrangement } \\
\text { with } \\
\text { different } \\
\text { interpolation } \\
\text { method for } \\
\text { STBC based }\end{array}$ \\
\hline
\end{tabular}

\begin{tabular}{|c|c|c|c|}
\hline & $\begin{array}{l}\text { OFDM } \\
\text { System } \\
\text { over } \\
\text { Frequency } \\
\text { Selective } \\
\text { Channel }\end{array}$ & & $\begin{array}{l}\text { OFDM } \\
\text { system is } \\
\text { Investigated } \\
\text { over } \\
\text { multipath } \\
\text { fading } \\
\text { channel. }\end{array}$ \\
\hline [4] & $\begin{array}{l}\text { Blind } \\
\text { STBC } \\
\text { Identificatio } \\
\mathrm{n} \quad \text { for } \\
\text { Multiple- } \\
\text { Antenna(M } \\
\text { A) OFDM } \\
\text { Systems }\end{array}$ & $\begin{array}{l}\text { IEEE } \\
\text { TRANSACTIONS } \\
\text { ON } \\
\text { COMMUNICATIO } \\
\text { NS, } \\
\text { May } 2014\end{array}$ & $\begin{array}{l}\text { A novel } \\
\text { STBC- } \\
\text { OFDM } \\
\text { identificatio } \\
\mathrm{n} \text { method is } \\
\text { proposed. }\end{array}$ \\
\hline [7] & $\begin{array}{l}\text { Training- } \\
\text { Based } \\
\text { MIMO } \\
\text { Channel } \\
\text { Estimation } \\
\text { A Study } \\
\text { of } \\
\text { Estimator } \\
\text { Tradeoffs } \\
\text { and } \\
\text { Optimal } \\
\text { Training } \\
\text { Signals }\end{array}$ & $\begin{array}{l}\text { IEEE } \\
\text { TRANSACTIONS } \\
\text { ON SIGNAL } \\
\text { PROCESSING, } \\
\text { MARCH } 2006\end{array}$ & $\begin{array}{l}\text { The } \\
\text { performance } \\
\text { of several } \\
\text { training- } \\
\text { based } \\
\text { MIMO } \\
\text { channel } \\
\text { estimation is } \\
\text { realize }\end{array}$ \\
\hline
\end{tabular}

\section{METHOD}

\subsection{STBC Based OFDM System Model}

The system model for STBC-OFDM with two transmission antenna and a receive antenna is shown in the Fig1. on the other hand, the system model can be extended to any no.of transmitting and receiving antennas to obtain high transmit diversity. At the transmitter end, the data sequence is generate and modulate according to any definite modulation scheme such as QPSK, BPSK or 16QAM. After this the output modulated data is passed through STBC encoder. The STBC encoder use to change the single input modulated information data into two parallel encoded output data by using Alamouti STBC scheme. The pilot sequence is also generated and modulates similarly to the data sequence. After that both encoded data and pilot subcarrier passed by the serial to parallel converter and finally reached at the (IFFT) Inverse Fast Fourier Transformation block. The output of the IFFT block expressed as

$$
\begin{aligned}
& x_{t, n}^{\beta}=\sum_{k=0}^{N-1} X_{t, k}^{\beta} e^{j 2 \pi k n / N} \\
& \beta=1,2, \quad n, k=0,1,2, \ldots . N-1
\end{aligned}
$$

Where data vector denotes by $X_{t, k}^{\beta}, x_{t, n}^{\beta}$ before and after of the IFFT block, $\beta$ denote the transmitting antenna 
index, and $\mathrm{n}, \mathrm{k}$ represents the $\mathrm{k}^{\text {th }}$ subcarrier and the its $\mathrm{n}^{\text {th }}$ time instant at the $\mathrm{t}^{\text {th }}$ symbol period. Where $\mathrm{N}$ denote the total number of OFDM data subcarrier. at last, the resulting signals are transmitted from the antennas following insertion of the cyclic prefix (CP) which is assumed to be largas compare to the delay spread of the multipath channel to avoid inter symbol interferences (ISI).The channel is assumed to be static or quasi-static for two time slot of STBC block. The channel model use in this paper is describe latter in this segment. The received signal can be obtain by taking the difficulty of transmitted data signal with the channel impulse reaction and can be expressed in term as

$$
r_{j, t, n}=\sum_{l=0}^{L-1} h_{j, t, l}^{\beta} x_{t, n-l}^{\beta}+w_{j, t, n}
$$

Where $h_{j, t, l}^{\beta}$ is the channel impulse response of the $l^{\text {th }}$ path at $t^{\text {th }}$ time slot for $\beta^{\text {th }}$ transmit antenna and $j^{\text {th }}$

Receive antenna in time domain. At the receiver end at first the $\mathrm{CP}$ is removed and then the received signal is processed by a Fast Fourier Transformation The FFT output of the received signal after the removal of $\mathrm{CP}$ can be written as

$$
\begin{aligned}
R_{j, t, k}= & \sum_{\beta=0}^{N_{t}} H_{j, t, k}^{\beta} X_{t, k}^{\beta}+W_{j, t, k} \\
& \text { Where } H_{j, t, k}^{\beta}=\sum_{k=0}^{N-1} h_{j, t, l}^{\beta} e^{-j 2 \pi 2 \pi / N}
\end{aligned}
$$

And $\mathrm{j}=1,2 \ldots \mathrm{Nr}, \beta=1,2$.

$H_{j, t, k}^{\beta}$ denote the frequency response of the channel from $\beta^{\text {th }}$ transmitting antenna to the $j^{\text {th }}$ receiving antenna and on the $k^{\text {th }}$ subcarrier. $\mathrm{N}_{\mathrm{t}}$ and $\mathrm{N}_{\mathrm{r}}$ represent the quantity of transmit and receive antennas. $W_{j, t, k}$ Is the additive white Gaussian noise with zero mean and unit variance. After the processed signal is decoded by the STBC decoding method. to conclude, the transmitted signal is recovers after taking the hard resolution of the decoded signal. In this paper, IEEE802.11 model with exponential power delay profile is adopted. The channel is modeled as finite impulse response with total L+1 non-zero path and with zero mean and average power of $\sigma_{1}^{2}$. The channel can be expressed as

$h_{1}=N\left(0, \frac{\sigma_{1}}{2}\right)+j N\left(0, \frac{\sigma_{1}}{2}\right)$

Where $N\left(0, \frac{\sigma_{1}}{2}\right)$ is zero mean with variance $\sigma_{1}^{2}$

power of the multipath component decreases exponentially. The first path of the model is choose to be

$$
\sigma_{0}^{2}=\frac{1-\lambda}{1-\lambda^{L+1}}
$$

Where $\lambda=e^{\frac{T_{S}}{\tau_{r m s}}}$ and $L=\frac{10 \tau_{r m s}}{T_{s}}$ The $T s$ and $\tau_{r m s}$ are the root mean and sampling period squared delay of the channel respectively. The energy of $l^{\text {th }}$ path can be written like

$$
\sigma_{l}^{2}=\sigma_{0}^{2} \lambda^{l}
$$

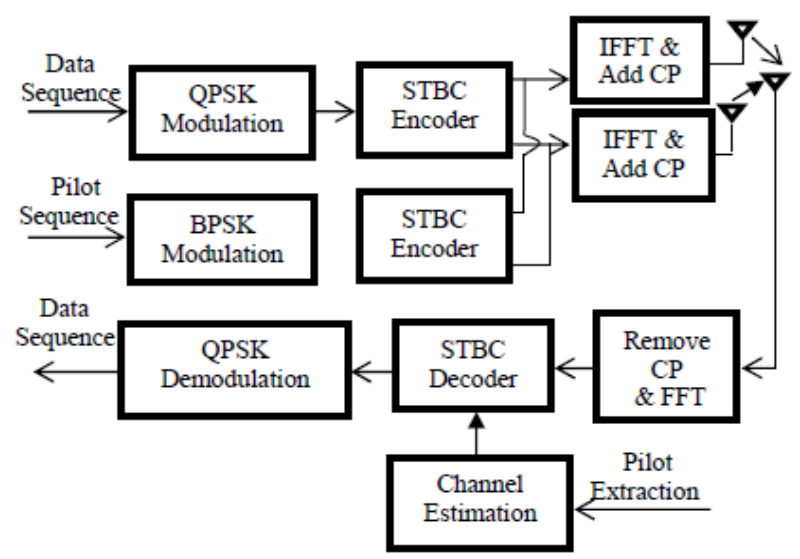

Figure 1 Block diagram of an STBC-OFDM system model.

\section{CONCLUSION}

In this paper, the pilot aided channel estimation technique by using comb type pilot arrangement with differ interpolation technique for STBC based OFDM scheme is investigated over multipath fading channel. The orthogonal properties of pilot arrangement have been described in a large detail. Couse to the orthogonal pilot arrangement in the two transmitting antennas, the channel estimation technique is simple and low computation cost because no matrix inversion is necessary. The simulation results show the comb type pilot with spline and low-pass interpolation technique of STBC-OFDM scheme performs better than the usual OFDM system. From the simulation result, clear that the low-pass interpolation outperform than spline type interpolation process. We can see that SER decrease as number of receiving antenna increases. The developed channel estimation has a failure of $2 \mathrm{~dB}$ to $2.5 \mathrm{~dB}$ SNR as compared to the model case where it is assumed that the perfect channel knowledge is known at the receiving end. So that from the simulation results and theory, it is proved that developed channel estimation technique is best suitable for the channel estimation purpose for multipath fading channel..

\section{REFERENCES}

[1] Emna Ben Slimane, Slaheddine larboui, Zouhed Ben Mabrouk, Ammar Bouallegue, " Pilot Assisted Channel Estimation in MIMO-STBC Systems Over TimeVarying Fading Channels" The 2014 International Workshop on Resource Allocation, Cooperation and Competition in Wireless Networks 978-3-901 882-63-0/1 4/\$31.00 @2014 IEEE.

[2] Duo Zhang, Guo Wei, Jinkang Zhu, Zhi Tian, “ On the Bounds of Feedback Rates for Pilot-Assisted MIMO Systems" IEEE TRANSACTIONS ON VEHICULAR TECHNOLOGY, VOL. 56, NO. 4, JULY 2007.

[3] Jyoti P. Patra, Poonam Singh, " A Comb-Type Pilot Symbol Aided Channel Estimation for STBC based 
OFDM System over Frequency Selective Channel" 9781-4799-2751-7/13/\$31.00 Â@2013 IEEE

[4] Mohamed Marey, Octavia A. Dobre, Robert Inkol, "Blind STBC Identification for Multiple-Antenna OFDM Systems" IEEE TRANSACTIONS ON COMMUNICATIONS, VOL. 62, NO. 5, MAY 2014 0090-6778/14\$31 2014 IEEE

[5] Leandro D' Orazio, Claudio Sacchi, Massimo Donelli, Francesco G.B. De Natale, “ MMSE Multi-User Detection with GA-assistedchannel Estimation for STBC MC-CDMA Mobile Communication Systems" 978-14244-2204-3/08/\$25.00 @2008 IEEE

[6] V. Tarokh, H. Jafarkhani, and A. R. Calderbank, "Space-time blockcodes from orthogonal designs," IEEE Trans. Inf. Theory, vol. 45, no. 5, pp. 1456-1467, Jul. 1999.

[7] M. Biguesh and A. B. Gershman, 'Training-based MIMO channel estimation: A study of estimator tradeofls and optimal training signals," IEEE Trans. Signal Process., vol. 54, no. 3, pp. 884-893, Mar. 2006.

[8] J.K. Cavers, "An Analysis of Pilot Symbol Assisted Modulation for Rayleigh Fading Channels," IEEE Trans. on Veh. Tech., vol. 40, no.4, pp. 686--693, Nov. 1991.S. Ohno and G. B. Giannakis, "Average-rate optimal PSAM transmissions over time-selective fading channels," IEEE Trans.Wireless Commun., vol. I, no. 4, pp. 712-720, Oct. 2002.

[9] K. Yu, 1. Evans, and 1. Collings, "Performance analysis of pilot symbol aided QAM for Rayleigh fading channels," in Proc. IEEE ICC, New York, NY, pp. 17311735, May 2002.

[10] S.S. Ikki, S. AI-Dharrab and M. Uysal, "Error Probability of OF Relaying with Pilot-Assisted Channel Estimation over Time-Varying Fading Channels," IEEE Transactions on Vehicular Technology, vol.61,no. I,393 397,2012 .
[11] Wenyu Li, Yunfei Chen and Norman C. Beaulieu, "BER Optimization of Pilot Symbol Assisted MRC PSI( for Slow Fading Channels " IEEE Communications Letters, vol. 13, no. 12, December 2009.

[12] Eiji Okamoto, Huan-Bang Li, and Tetsushi Ikegami, "A Pilot Symbol Assisted Compensation Scheme of Fading and Frequency Offset for 16QAM," IEEE International Conference on Universal Personal Communications (ICUPC), vol. 2, pp. 921 - 924, 1998.

[13] Xiaodong Cai and Georgios B. Giannakis, "Adaptive PSAM Accounting for Channel Estimation and Prediction Errors," IEEE Transactions on Wireless Communications, vol. 4, no. 1, January 2005.

[14] Amine Maaref and Sonia Alssa, "Optimized RateAdaptive PSAM for MIMO MRC Systems with Transmit and Receive CSI Imperfections," IEEE Transactions on Communications, vol. 57, no. 3, March 2009.

[15] Wenyu Li and Norman C. Beaulieu, "Effects of ChannelEstimation Errors on Receiver Selection-Combining Schemes for Alamouti MIMO Systems With BPSI(," IEEE Transactions on Communications, vol. 54, no. I, January 2006.

[16] Jie Wu and Gary J. Saulnier, "Orthogonal Space-Time Block Code Over Time-Varying Flat-Fading Channels: Channel Estimation, Detection, and Performance Analysis," IEEE Transactions on Communications, vol. 55, no. 5, May 2007.

[17] Duo Zhang, Guo Wei, Jinkang Zhu and Zhi Tian, "On the Bounds of Feedback Rates for Pilot-Assisted MIMO Systems," IEEE Transactions on Vehicular Technology, vol. 56, vo. 4, July 2007 .

[18] H. Meyr, M. Moenec1aey, and S. A. Fechte1. "Digital Communication Receivers," New York: Wiley, 1997. 